\title{
Synthesis of New Isoxazolin - Phenoxathiin Derivatives
}

\author{
Ayad Ahmed Mohamad"and Suad M. AL-Araji** \\ * Department of Research and Quality Control, Daura Refinery, Ministry of Oil. \\ *** Department of Chemistry, College of Science, University of Baghdad, Baghdad-Iraq.
}

\begin{abstract}
The aim of the present work is synthesis of new phenoxathiin derivatives. The 2-(oxoalken1-yl) phenoxathiin derivatives (3a-3j) obtained from the reaction 2-acetylphenoxathiin with different aromatic aldehyde in the presence of sodium hydroxide. The reaction of 2-(oxoalken-1-yl) phenoxathiin derivatives (3a-3j) with hydroxylamine hydrochloride in ethanolic sodium hydroxide solution to get 2-(isoxazolin-3-yl) phenoxathiin derivatives (4a-4j) which substituted at position (5) in isoxazoline ring with different aryl groups according to aromatic aldehyde used in the preparation of (3a-3j). All the synthesized compounds were characterized by different identification techniques to confirm structure which has been obtained.
\end{abstract}

Keywords: Phenoxathiin, Oxoalken derivatives,Hydroxylamine,Isoxazoline.

\section{Introduction}

Phenoxathiin is given as the preferred name by Patterson and Capell [1]. The method of preparation for phenoxathiin has been used most widely encountered is the reaction between diphenyl ether and sulfur in the presence of anhydrous aluminum chloride $[2,3]$. Large number has been proposed for phenoxathiin and some of its derivatives, recently addition of phenoxathiincation radical to a cyclic alkene in acetonitril $(\mathrm{MeCN})$ solution occurred stereo specifically to form bis(10-phenoxathiiniumyl) alkane adducts [4]. Phenoxathiin derivatives have recently gained attention owing to their fluorescent properties [5].Ionescuand Popovici [6], studied the emission properties of 2-phenoxathiinyl-5phenyloxazole and 5-phenoxathiinyl-2phenyloxazole derivatives by measuring the absorption and emission spectra of the derivatives above in cyclohexane and methanol. Aly and Wasfy[7], describedthat [6(phenoxathiin-2-yl)

-2,3,4,5-tetrahydropyridazine-3-one] was successively synthesized by the condensation of [4-(phenoxathiin-2-yl)-4-oxobutanoic acid] with hydrazine hydrate in boiling ethanol. Polyamides with inherent viscosities in the range of (0.5-2.9) were readily prepared by the polycondensations of phenoxathiindiamines with aromatic diacyl chlorides and aromatic diamines with new phenoxathiindiacyl chlorides[8].Tintaru, Hillebrand andThevand [9], studied the inclusion complexes of the forms of 3-carboxy-(1) and 2-carboxyphenoxathiin (2) with $\beta$-cyclodextrin by both one- and two-dimensional NMR spectroscopy[10]. Miguel Yus [11] described that phenoxathiin was lithiated using 4,4'-ditert-butylbiphenyl (DTBB) as the catalyst in $\mathrm{THF}$ at $-78^{\circ} \mathrm{C}$, so intermediate was obtained by a carbon-sulfur reductive cleavage. This specie reacted with electrophiles giving, after hydrolysis with $(3 \mathrm{M})$ hydrochloric acid, the corresponding compound.

\section{Experimental}

FT-IR spectra were recorded on [SHIMADZU] FT-IR 8400s spectrophotometer; Solid samples were run in $\mathrm{KBr}$ disc, Liquid were run as smears. UV spectra were recorded on UV-Visible Spectrophotometer [SHIMADZU] UV160A. ${ }^{1} \mathrm{H}-\mathrm{NMR}$ spectra were recorded on ultra shield $300 \mathrm{MHz}$ spectrophotometer in acetone$\mathrm{d}_{6}$ solutions and withwithtetramethylsilane as internal standard. Melting points were determined in a [GallenKamp] melting point apparatus with sample contained in open capillary glass tube in an electrically heated metal block apparatus.

\section{General procedure for synthesis of phenoxathiinand itsderivatives}

\section{Phenoxathiin (1) $[12,13]$}

A mixture of $188.6 \mathrm{~g}$. (1.1 mol) of phenyl ether, $25.6 \mathrm{~g}$. of sulfur and $51.0 \mathrm{~g}$. $(0.38 \mathrm{~mol})$ of anhydrous aluminum chloride, maintained on steam bath for $4 \mathrm{hrs}$. The reaction mixture 
was poured slowly, with stirring, into ice bath to which $(25 \mathrm{ml}$.) of concentrated hydrochloric acid was added. After the two layers were separated the water layer was discarded and the (phenyl ether-phenoxathiin) layer dried overnight with calcium chloride, this mixture was distilled under reduced pressure from a 500-ml specialClaisen flask. After removal of the phenyl ether the fraction boiling at (140-160 ${ }^{\circ} \mathrm{C} / 5 \mathrm{~mm}$.), phenoxathiin was collected at $\left(150-152^{\circ} \mathrm{C} / 5 \mathrm{~mm}\right.$.). The product was recrystallized from methyl alcohol, m.p. $(56-57)^{\circ} \mathrm{C}$,yield $80 \%$.

\section{2-acetylphenoxathiin (2) [14]}

A mixture of $(22.9 \mathrm{~g}, 0.114 \mathrm{~mol})$ phenoxathiin, (9.7 g, $0.155 \mathrm{~mol}, 8.8 \mathrm{ml}$ ) acetyl chloride and carbon disulphide $(120 \mathrm{ml})$ was stirred while anhydrous aluminum chloride $(15.5 \mathrm{~g}, 0.116 \mathrm{~mol})$ was added in small portions. The red mixture was stirred for ( $2 \mathrm{hrs}$.) at room temperature and refluxed on the water bath for a further ( 24 hours), the mixture was cooled then it poured to a mixture of ice and hydrochloric acid, the product was recrystallized once from ethanol and twice from petroleum ether b.p. $(80-100)^{\circ} \mathrm{C}$, m.p. $112^{\circ} \mathrm{C}$, yield $52.5 \%$.

\section{2-(oxoalken-1-yl) phenoxathiin derivatives (3a-3j) [15]}

A mixture of $(3 \mathrm{~g}, \quad 0.013 \mathrm{~mol}) \quad 2$ acetylphenoxathiin and $(1.56 \mathrm{~g}, 0.0147 \mathrm{~mol})$ of appropriate benzaldehyde in $(80 \mathrm{ml})$ of ethanol and $(1.5 \mathrm{ml})$ of $(1 \% \mathrm{NaOH})$ solution was refluxed for $(2 \mathrm{hrs})$. The reaction mixture was poured into cold water, the precipitate filtered off and recrystallized from (ethanol-water) (3:1) to give $(3 a-3 j)$. Table (1) represent the physical data of compounds (3a$3 \mathrm{j})$.Characteristic bands of FT-IR spectra of compounds (3a-3j) are listed in Table (2).

\section{2-(5-phenylisoxazolin-3-yl) phenoxathiin derivatives $(\mathbf{4 a - 4 j})[\mathbf{1 6}, \mathbf{1 7}]$}

A solution of $(0.33 \mathrm{~g}, 0.001 \mathrm{~mol})$ of 2(3-phenyl-1-oxypropen-1-yl) phenoxathiin (3a) and $(0.07 \mathrm{~g}, 0.001 \mathrm{~mol})$ of hydroxylamine hydrochloride in ethanolic sodium hydroxide solution $(0.01 \mathrm{~mol})$ was refluxed for $(6 \mathrm{hrs})$. By concentration and cooling the product separated out, recrystallized using (ethanolwater) $(3: 1)$ to give $(4 a-4 j)$ derivatives. Table (3) represent the physical data of compounds (4a-4j). Characteristic bands of FT-IR spectra of compounds $(4 \mathrm{a}-4 \mathrm{j})$ are listed in Table (4).

\section{Results and Discussion}

Phenoxathiin(1) was obtained from diphenyl ether and sulfur reacted in the presence of aluminum chloride, FT-IR spectrum of phenoxathiin showed a stretching band at $3063 \mathrm{~cm}^{-1}$ for aromatic $(\mathrm{C}-\mathrm{H})$, $1590 \mathrm{~cm}^{-1}$ and $1450 \mathrm{~cm}^{-1}$ assigned to the aromatic system $(\mathrm{C}=\mathrm{C})$ str., $1219 \mathrm{~cm}^{-1}$ and $1026 \mathrm{~cm}^{-1}$ assigned to asym. and sym.(C-OC)str. The ${ }^{1} \mathrm{H}-\mathrm{NMR}$ spectrum showed signals between $\delta(6.8-7.3) \mathrm{ppm}$ assigned to aromatic protons. U.V spectrum showed an absorption $\lambda_{\max }$ at $252 \mathrm{~nm}$ due to $\left(\pi \rightarrow \pi^{*}\right)$ electronic transitions. 2-acetyl phenoxathiin (2) was prepared from reaction of phenoxathiin with acetyl chloride in dry carbon disulfide in presence of anhydrous aluminum chloride to gave compound (2) through Friedel Crafts acylation method [16].FT-IR spectrum of compound (2) showed weak bands at $3078 \mathrm{~cm}^{-1}$ for aromatic (C-H) str., $2962 \mathrm{~cm}^{-1}$, $2931 \mathrm{~cm}^{-1}$ and $2877 \mathrm{~cm}^{-1}$ aliphatic (C-H)str. of $\left(\mathrm{CH}_{3}\right)$ acetyl group, strong bands at $1674 \mathrm{~cm}^{-1}$ $(\mathrm{C}=\mathrm{O})$ str., two bands at $1558 \mathrm{~cm}^{-1}$ and 1465 $\mathrm{cm}^{-1}$ aromatic system $(\mathrm{C}=\mathrm{C})$ str. and $756 \mathrm{~cm}^{-1}$ $(--\mathrm{C}-\mathrm{H})$ aromatic ring. The ${ }^{1} \mathrm{H}-\mathrm{NMR}$ spectrum showed a signal at $\delta 2.6 \mathrm{ppm}$ assigned to the three protons of the acetyl group and signals between $\delta(7.0-7.3) \mathrm{ppm}$ assigned to aromatic protons. Through nucleophilic addition reaction of compound (2) to aldehydes and ketones, compound (2) undergoes the characteristic condensation reaction with different kinds of aromatic aldehydes in ethanol instead of $1 \% \mathrm{NaOH}$ solution as a catalyst to afford (3a-3j) derivatives. The FT-IR spectra of compounds (3a-3j) showed absorption bands at (16741681) $\mathrm{cm}^{-1}(\mathrm{C}=\mathrm{O})$ str., and $(1600-1608) \mathrm{cm}^{-1}$ aliphatic $(\mathrm{C}=\mathrm{C})$ str. The ${ }^{1} \mathrm{H}-\mathrm{NMR}$ spectrum showed a signal at $\delta 2.6 \mathrm{ppm}$ assigned to aliphatic three protons of methoxy group, signals between $\delta(7.0-7.3) \mathrm{ppm}$ assigned to both olefinic $(\mathrm{H} 1)$ and $(\mathrm{H} 2)$ respectively and a signals at $\delta 7.5 \mathrm{ppm}$ and $\delta 7.9 \mathrm{ppm}$ assigned to aromatic protons. Table (1) represent the physical data of compounds (3a-3j). Characteristic absorption bands of FT-IR and U.V spectra of compounds (3a-3j) are listed in 
Table (2). The additive property of the exocyclic $(\mathrm{C}=\mathrm{C})$ in (3) conjugated with the carbonyl group promoted us to investigate their behavior towards the action of hydroxylamine react with (3) in the presence of ethanolic sodium hydroxide solution giving 5-phenyl isoxazoline $(4 \mathrm{a}-4 \mathrm{j})$ as shown in the Scheme. The structure of these compounds were established from FT-IR and U.V.FT-IR spectrum showed reactivity medium weak bands at $3063 \quad \mathrm{~cm}^{-1}$ and $3062 \mathrm{~cm}^{-1}$ aromatic (C-H)str, $1681 \mathrm{~cm}^{-1}$ and $1674 \mathrm{~cm}^{-1}$ imine $(\mathrm{C}=\mathrm{N})$ str.and bands between (817-825) $\mathrm{cm}^{-1}(\mathrm{~N}-\mathrm{O})$ str.U.V. spectrum showed an absorption $\lambda_{\max }$ at $252 \mathrm{~nm}$ due to $\left(\pi \rightarrow \pi^{*}\right)$ electronic transitions. The ${ }^{1} \mathrm{H}-\mathrm{NMR}$ spectra for (4e) showed a strong singlet signal at $\delta 2.6 \mathrm{ppm}$ which was assigned to the three protons of the methoxy group. signals at $\delta 2.3 \mathrm{ppm}$ and $\delta 3.9 \mathrm{ppm}$ assigned to aliphatic two protons $(\mathrm{H} 4)$ and proton $(\mathrm{H} 5)$ of the isoxazoline ring respectively. Finally, signals between $\delta(7.1-7.8)$ ppm for aromatic protons. Table (3) represent the physical data of compounds $(4 \mathrm{a}-4 \mathrm{j})$. Characteristic absorption bands of FT-IR and U.V spectra of compounds $(4 a-4 j)$ are listed in Table (4). Table (5) representthe ${ }^{1} \mathrm{H}-\mathrm{NMR}$ spectra for compounds (1, 2, 3e, 3h, 4eand 4j). In addition, Figs. (1, 3, 4) show FT-IR spectra for prepared comps. (2, 3a, 3f), respectively. Also, Figs. (2, 5-8) show H-NMR spectra for prepared comps. $(2,3 \mathrm{e}$, $3 \mathrm{~h}, 4 \mathrm{e}, 4 \mathrm{j}$ ), respectively.

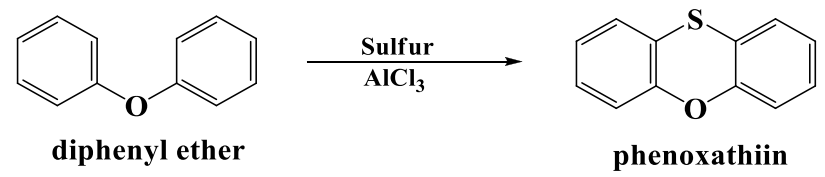

(1)

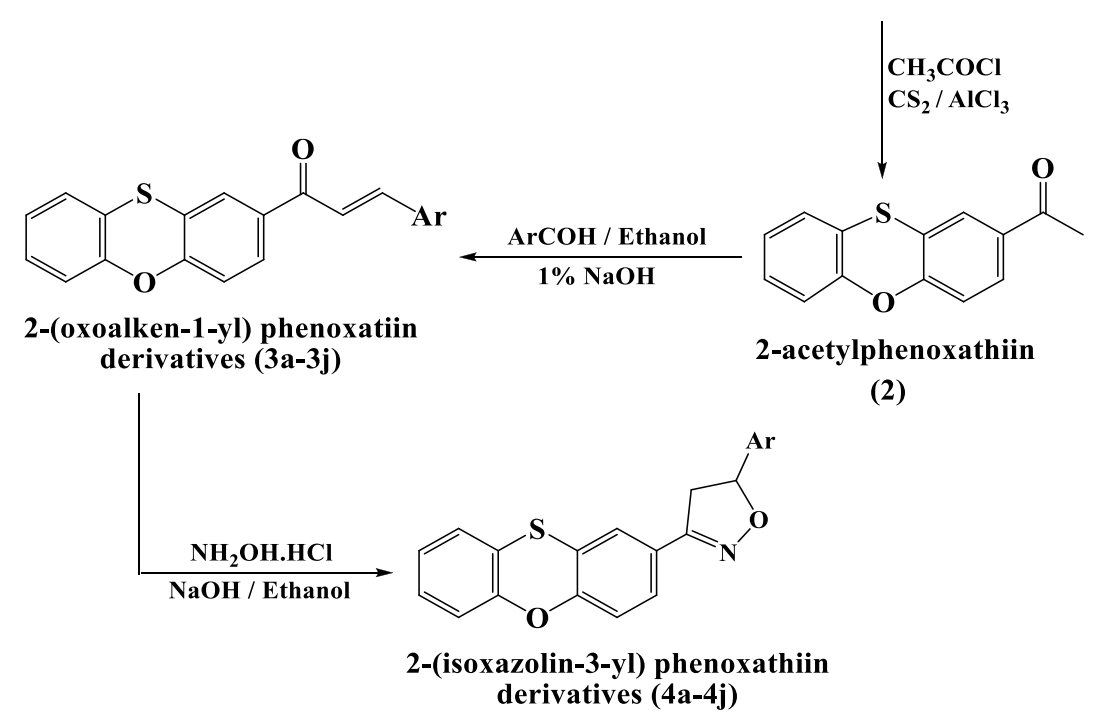

Where (Ar) is

a )

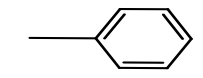

d )
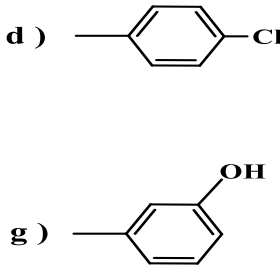

b )

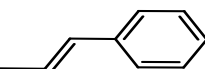

c )<smiles>Cc1cccc([N+](=O)[O-])c1</smiles>

e )<smiles>COC1=C(C)CCC=C1</smiles>

f)<smiles>Cc1ccc(O)cc1</smiles>

h )<smiles>Cc1ccc(Br)cc1</smiles>

i )<smiles>Cc1c(O)ccc2ccccc12</smiles>

j )<smiles>Cc1ccc(O)c(O)c1</smiles> 
Table (1)

Represent the physical data of compounds (3a-3j).

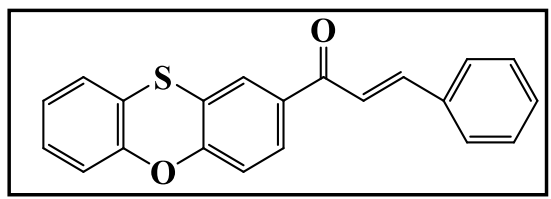

\begin{tabular}{|c|c|c|c|c|c|}
\hline $\begin{array}{l}\text { Comp. } \\
\text { No. }\end{array}$ & Scientific name & m.p. ${ }^{\circ} \mathrm{C}$ & $\begin{array}{l}\text { Yield } \\
\%\end{array}$ & $\begin{array}{l}\text { Color of } \\
\text { crystal }\end{array}$ & Chemistry structure \\
\hline $3 a$ & $\begin{array}{l}\text { 2-(3-phenyl-1-oxypropen-1- } \\
\text { yl) phenoxathiin }\end{array}$ & $100-102$ & 73.0 & Yellowish & \\
\hline $3 b$ & $\begin{array}{l}\text { 2-(5-phenyl-1-oxypentadien- } \\
\text { 1-yl) phenoxathiin }\end{array}$ & $102-104$ & 53.0 & $\begin{array}{l}\text { Light- } \\
\text { yellow }\end{array}$ & \\
\hline $3 c$ & $\begin{array}{l}\text { 2-[3-(3-nitrophenyl)-1- } \\
\text { oxypropen-1-yl] } \\
\text { phenoxathiin }\end{array}$ & $92-94$ & 65.0 & Yellow & \\
\hline $3 d$ & $\begin{array}{l}\text { 2-[3-(4-chlorophenyl)-1- } \\
\text { oxypropen-1-yl] } \\
\text { phenoxathiin }\end{array}$ & $94-96$ & 45.2 & $\begin{array}{l}\text { Deep- } \\
\text { yellow }\end{array}$ & \\
\hline $3 e$ & $\begin{array}{l}\text { 2-[3-(2-methoxyphenyl)-1- } \\
\text { oxypropen-1-yl] } \\
\text { phenoxathiin }\end{array}$ & $96-98$ & 60.0 & $\begin{array}{l}\text { Deep- } \\
\text { yellow }\end{array}$ & \\
\hline $3 f$ & $\begin{array}{l}\text { 2-[3-(4-hydroxyphenyl)-1- } \\
\text { oxypropen-1-yl] } \\
\text { phenoxathiin }\end{array}$ & $103-105$ & 67.0 & Reddish & \\
\hline $3 g$ & $\begin{array}{l}\text { 2-[3-(3-hydroxyphenyl)-1- } \\
\text { oxypropen-1-yl]phenoxathiin }\end{array}$ & $102-104$ & 67.2 & $\begin{array}{l}\text { Yellow- } \\
\text { reddish }\end{array}$ & \\
\hline $3 \mathrm{~h}$ & $\begin{array}{l}\text { 2-[3-(4-bromophenyl)-1- } \\
\text { oxypropen-1-yl] } \\
\text { phenoxathiin }\end{array}$ & $106-108$ & 55.9 & Yellow & \\
\hline $3 i$ & $\begin{array}{l}\text { 2-[3-(2-hydroxy-1-naphthyl)- } \\
\text { 1-oxypropen-1-yl] } \\
\text { phenoxathiin }\end{array}$ & $92-94$ & 53.9 & Black & \\
\hline $3 \mathrm{j}$ & $\begin{array}{l}\text { 2-[3-(3,4-dihydroxyphenyl)- } \\
\text { 1-oxypropen-1-yl] } \\
\text { phenoxathiin }\end{array}$ & $93-95$ & 66.0 & Brown & \\
\hline
\end{tabular}


Table (2)

Infrared absorption data for compounds (3a-3j).

\begin{tabular}{|c|c|c|c|c|c|c|c|}
\hline \multirow{2}{*}{$\begin{array}{r}\text { Comp. } \\
\text { No. }\end{array}$} & \multirow[b]{2}{*}{ Chemistry structure } & \multicolumn{5}{|c|}{ FTIR spectral data $\mathrm{cm}^{-1}$} & \multirow{2}{*}{$\begin{array}{l}U . V . \\
\left(\lambda_{\max }\right) \\
n m\end{array}$} \\
\hline & & $v(C=O)$ & $\begin{array}{l}v(C-H) \\
\text { aromatic }\end{array}$ & $\begin{array}{l}v(C-H) \\
\text { olefinic }\end{array}$ & $v(C=C)$ & $\begin{array}{l}\text { Other } \\
\text { bands }\end{array}$ & \\
\hline $3 a$ & & 1680 & 3076 & 3018 & 1608 & - & $\begin{array}{l}249 \\
344\end{array}$ \\
\hline $3 b$ & & 1681 & 3090 & 3010 & 1600 & $\begin{array}{l}(\mathrm{C}-\mathrm{H}) \\
\text { olefinic } \\
3010\end{array}$ & 248 \\
\hline $3 c$ & & 1681 & 3070 & 2977 & 1600 & $\begin{array}{r}\left(\mathrm{NO}_{2}\right) \\
1535 \\
1350\end{array}$ & $\begin{array}{l}258 \\
300\end{array}$ \\
\hline $3 \mathrm{~d}$ & & 1674 & 3078 & 3009 & 1600 & $\begin{array}{r}(\mathrm{C}-\mathrm{Cl}) \\
1095\end{array}$ & 249 \\
\hline $3 e$ & & 1674 & 3078 & 3008 & 1600 & $\begin{array}{r}(\mathrm{C}-\mathrm{O}-\mathrm{C}) \\
1249 \\
1026\end{array}$ & $\begin{array}{l}250 \\
327\end{array}$ \\
\hline $3 \mathrm{f}$ & & 1674 & 3078 & 3009 & 1600 & $\begin{array}{r}(\mathrm{O}-\mathrm{H}) \\
3433\end{array}$ & 251 \\
\hline $3 \mathrm{~g}$ & & 1674 & 3075 & 3030 & 1600 & $\begin{array}{r}(\mathrm{O}-\mathrm{H}) \\
3440\end{array}$ & 248 \\
\hline $3 \mathrm{~h}$ & & 1674 & 3078 & 3009 & 1600 & $\begin{array}{r}(\mathrm{C}-\mathrm{Br}) \\
632\end{array}$ & 252 \\
\hline $3 \mathrm{i}$ & & 1674 & 3078 & 3008 & 1600 & $\begin{array}{r}(\mathrm{O}-\mathrm{H}) \\
3409\end{array}$ & 246 \\
\hline $3 \mathrm{j}$ & & 1674 & 3078 & 3009 & 1600 & $\begin{array}{r}(\mathrm{O}-\mathrm{H}) \\
3471\end{array}$ & $\begin{array}{l}254 \\
300 \\
360\end{array}$ \\
\hline
\end{tabular}




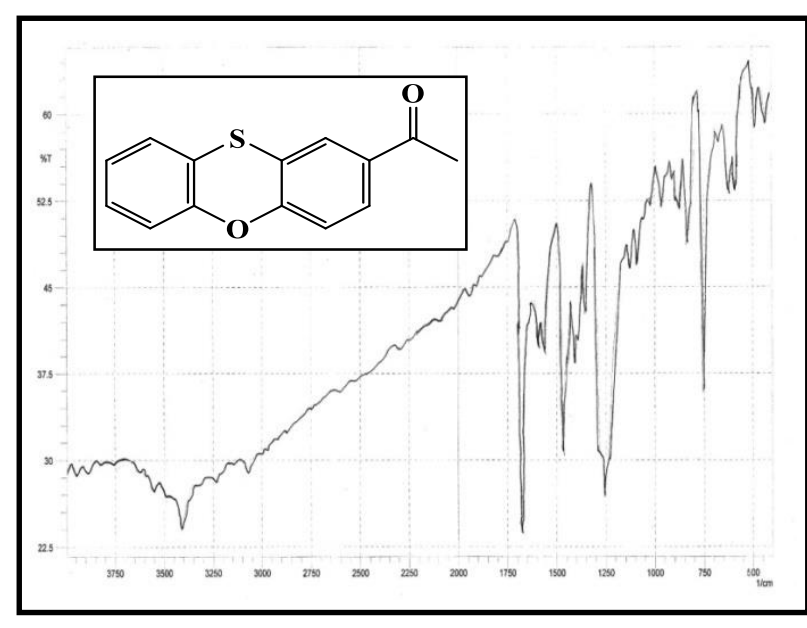

Fig.(1) FT-IR spectrum for compound (2).

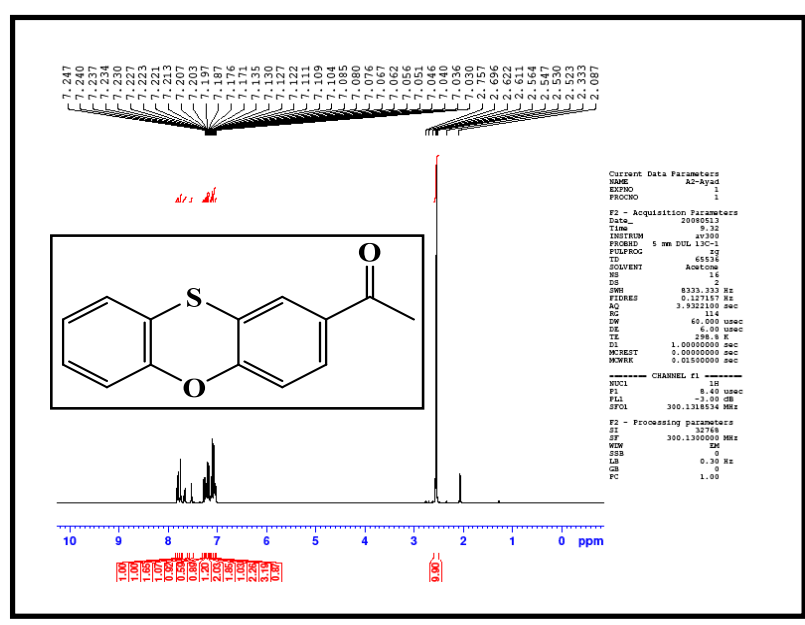

Fig.(2) ${ }^{l} \mathrm{H}$-NMR spectrum for compound (2).

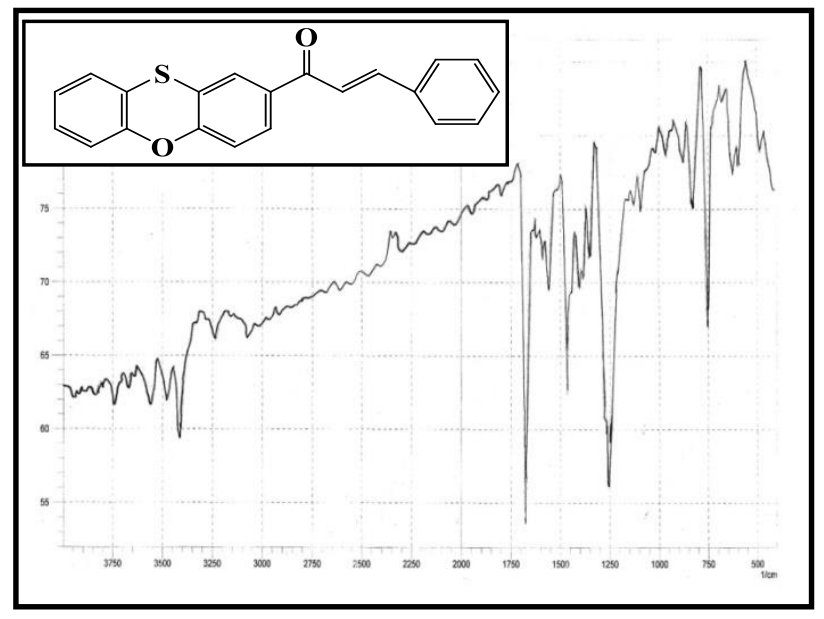

Fig.(3)FT-IR spectrum for compound (3a).

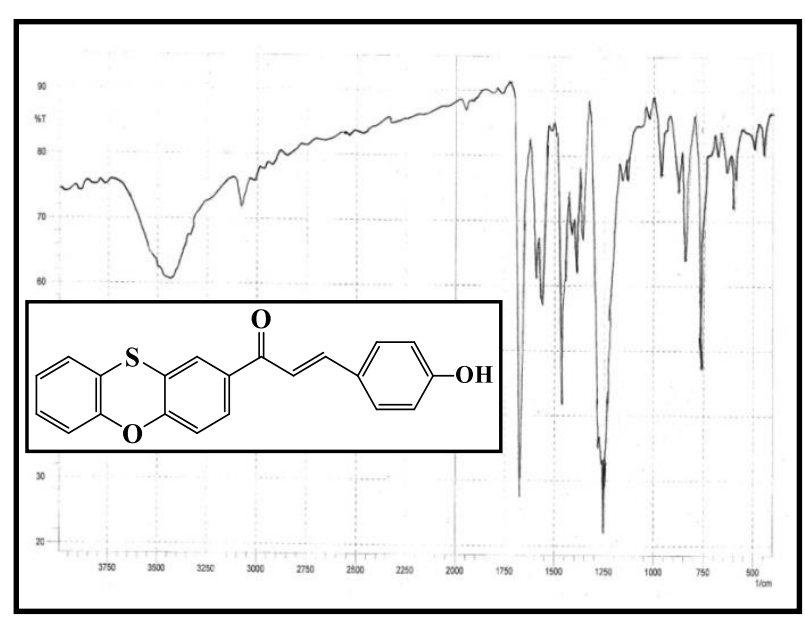

Fig.(4) FT-IR spectrum for compound (3f).

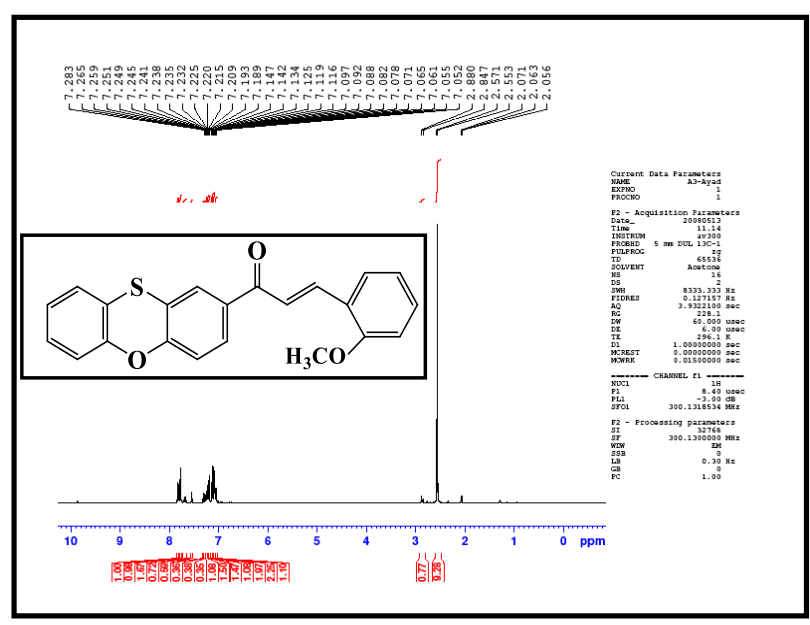

Fig.(5) ${ }^{1} \mathrm{H}$-NMR spectrum for compound $(3 e)$.

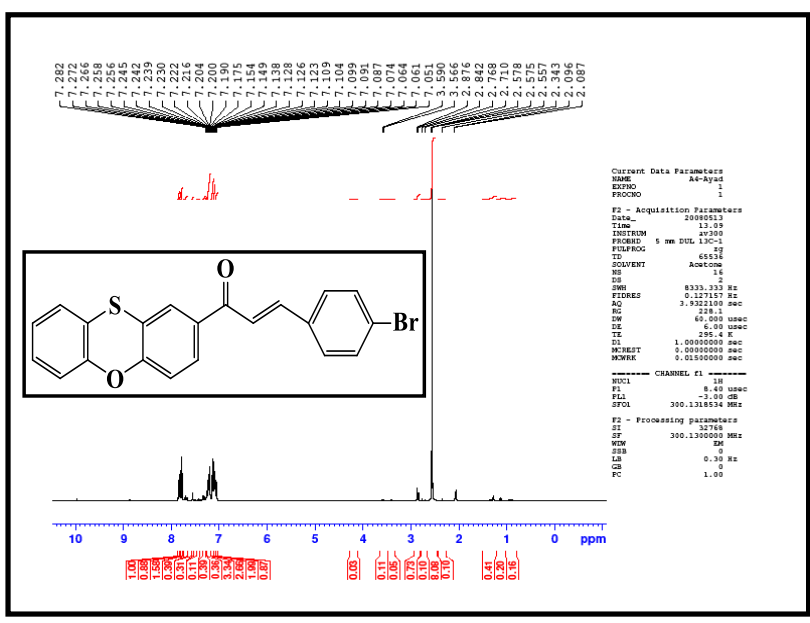

Fig.(6) ${ }^{1} \mathrm{H}$-NMR spectrum for compound

(3h). 
Table (3)

Represent the physical data of compounds (4a-4j).

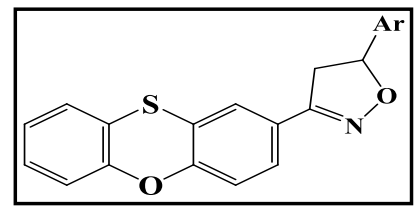

\begin{tabular}{|c|c|c|c|c|c|}
\hline $\begin{array}{r}\text { Comp. } \\
\text { No. }\end{array}$ & Scientific name & m.p. ${ }^{\circ} \mathrm{C}$ & Yield \% & $\begin{array}{r}\text { Color of } \\
\text { crystal }\end{array}$ & Chemistry structure \\
\hline $4 a$ & $\begin{array}{l}\text { 2-(5-phenylisoxazolin-3-yl) } \\
\text { phenoxathiin }\end{array}$ & $126-128$ & 60.0 & Yellowish & \\
\hline $4 \mathrm{~b}$ & $\begin{array}{l}\text { 2-(5-styrenylisoxazolin-3-yl) } \\
\text { phenoxathiin }\end{array}$ & $112-114$ & 50.0 & $\begin{array}{r}\text { Deep } \\
\text { yellow }\end{array}$ & \\
\hline $4 c$ & $\begin{array}{l}\text { 2-[5-(3-nitrophenyl) } \\
\text { isoxazolin-3-yl] } \\
\text { phenoxathiin }\end{array}$ & $98-100$ & 80.0 & Brown & \\
\hline $4 d$ & $\begin{array}{l}\text { 2-[5-(4-chlorophenyl) } \\
\text { isoxazolin-3-yl] } \\
\text { phenoxathiin }\end{array}$ & $96-98$ & 49.1 & Yellow & \\
\hline $4 e$ & $\begin{array}{l}\text { 2-[5-(2-methoxyphenyl) } \\
\text { isoxazolin-3-yl] } \\
\text { phenoxathiin }\end{array}$ & $128-130$ & 85.0 & $\begin{array}{r}\text { Deep } \\
\text { yellow }\end{array}$ & \\
\hline $4 f$ & $\begin{array}{l}\text { 2-[5-(4-hydroxyphenyl) } \\
\text { isoxazolin-3-yl] } \\
\text { phenoxathiin }\end{array}$ & $110-112$ & 47.6 & Yellow & \\
\hline $4 g$ & $\begin{array}{l}\text { 2-[5-(3-hydroxyphenyl) } \\
\text { isoxazolin-3-yl] } \\
\text { phenoxathiin }\end{array}$ & $105-107$ & 30.0 & Yellow & \\
\hline $4 \mathrm{~h}$ & $\begin{array}{l}\text { 2-[5-(4-bromophenyl) } \\
\text { isoxazolin-3-yl] } \\
\text { phenoxathiin }\end{array}$ & $115-117$ & 64.0 & Yellowish & \\
\hline $4 \mathrm{i}$ & $\begin{array}{l}\text { 2-[5-(2-hydroxynaphthyl) } \\
\text { isoxazolin-3-yl] } \\
\text { phenoxathiin }\end{array}$ & $143-145$ & 66.1 & Black & \\
\hline $4 j$ & $\begin{array}{l}\text { 2-[5-(3,4-dihydroxyphenyl) } \\
\text { isoxazolin-3-yl] } \\
\text { phenoxathiin }\end{array}$ & $165-167$ & 70.5 & Black & \\
\hline
\end{tabular}


Table (4)

Infrared absorption data for compounds (4a-4).

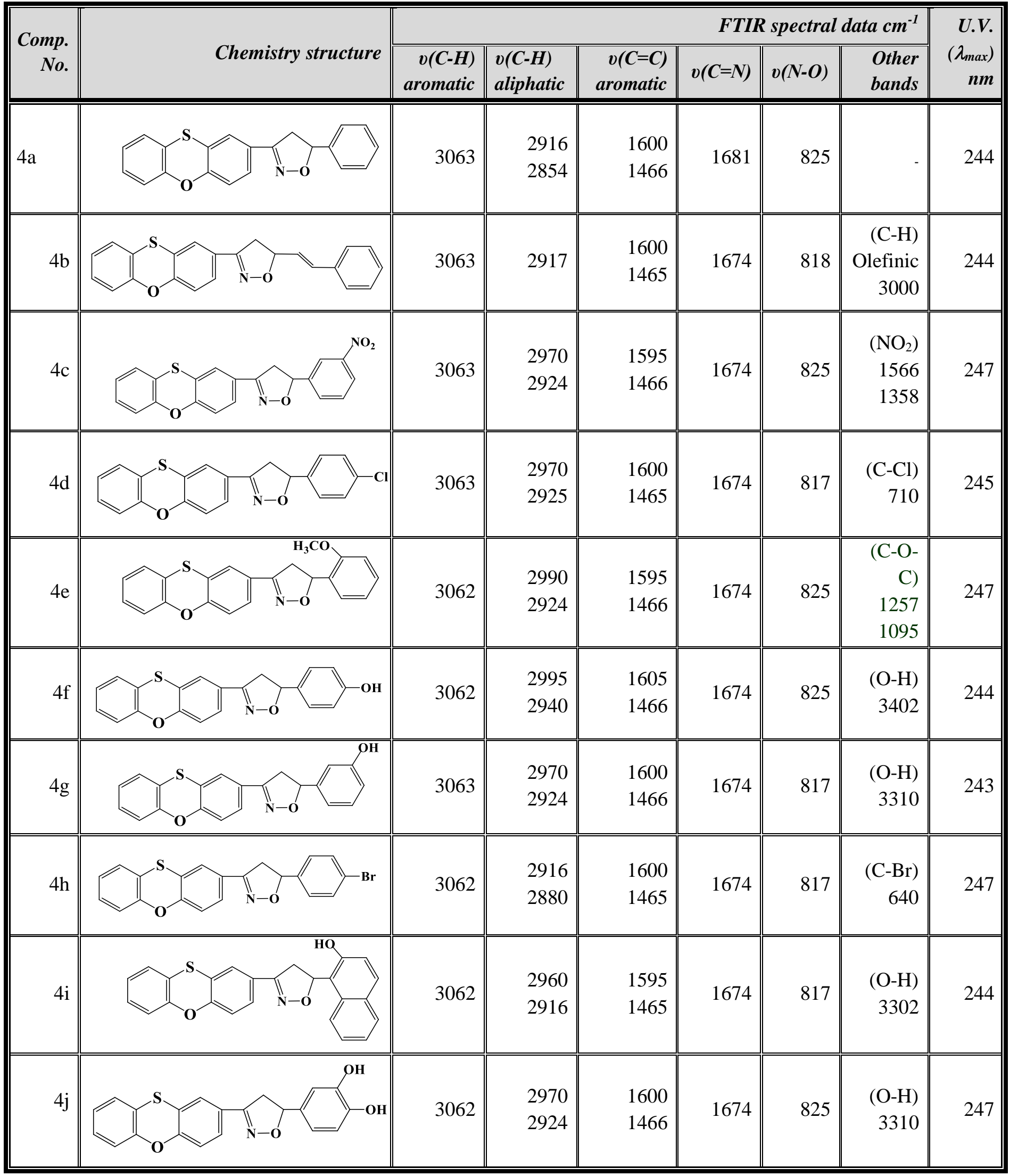




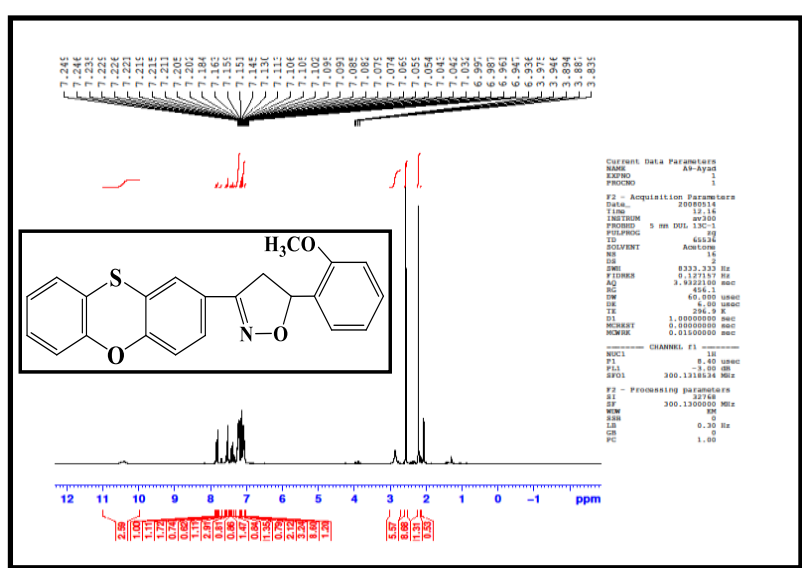

Fig.(7) ${ }^{1} \mathrm{H}$-NMR spectrum for compound $(4 e)$.

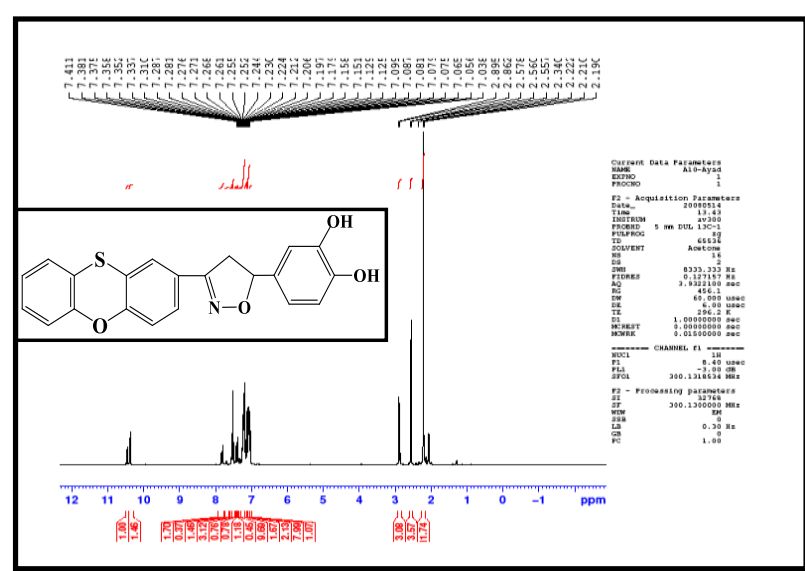

Fig.(8) ${ }^{1}$ H-NMR spectrum for compound (4j).

Table (5)

${ }^{1} \mathrm{H}$-NMR spectra for compounds $(1,2,3 e, 3 h, 4 e, 4 j)$.

\begin{tabular}{|c|c|c|c|}
\hline $\begin{array}{c}\text { Comp. } \\
\text { No. }\end{array}$ & Compound structure & $\delta H$ aromatic ppm & $\delta H$ other bands ppm \\
\hline 1 & & $(6.8-7.3)(\mathrm{m}, 8 \mathrm{H})$ & - \\
\hline 2 & & $(7.0-7.3)(m, 7 H)$ & $2.6(\mathrm{~s}, 3 \mathrm{H})$ acetyl protons \\
\hline $3 \mathbf{e}$ & & $(7.0-7.3)(\mathbf{m}, 11 H)$ & $\begin{array}{c}2.6(\mathrm{~s}, 3 \mathrm{H}) \text { methoxy protons } \\
{\left[\begin{array}{c}(\mathrm{s}, 1 \mathrm{H}) \mathrm{H} 1,7.9(\mathrm{~s}, 1 \mathrm{H}) \mathrm{H} 2] \\
\text { olefin protons }\end{array}\right.}\end{array}$ \\
\hline $3 \mathbf{h}$ & & $(7.0-7.3)(\mathrm{m}, 11 \mathrm{H})$ & $\begin{array}{c}{\left[\begin{array}{c}7.5(\mathrm{~s}, 1 \mathrm{H}) \mathrm{H} 1,7.8(\mathrm{~s}, 1 \mathrm{H}) \mathrm{H} 2] \\
\text { olefin protons }\end{array}\right.}\end{array}$ \\
\hline $4 e$ & & $(7.1-7.8)(\mathrm{m}, 11 \mathrm{H})$ & $\begin{array}{c}2.6(\mathrm{~s}, 3 \mathrm{H}) \text { methoxy protons } \\
{[2.3(\mathrm{~s}, 2 \mathrm{H}) \mathrm{H} 4,3.9} \\
(\mathrm{s}, 1 \mathrm{H}) \mathrm{H} 5] \\
\text { isoxazolin ring protons }\end{array}$ \\
\hline $4 \mathbf{j}$ & & $(7.0-7.8)(\mathrm{m}, 10 \mathrm{H})$ & $\begin{array}{l}\text { [2.6 }(\mathrm{s}, 2 \mathrm{H}) \mathrm{H} 4,2.9(\mathrm{~s}, 1 \mathrm{H}) \mathrm{H5}] \\
\text { isoxazolin ring protons } \\
10.4(\mathrm{~d}, 2 \mathrm{H}) \text { hydroxyl protons }\end{array}$ \\
\hline
\end{tabular}




\section{References}

[1] Patterson. A. M. and Capell. L. "The Ring Index". Reinhold Publishing Corporation, New York, 1940.

[2] Gilman. H, Ess. V, Marian. W, Willis. H. B. and Stuckwisch. C. G."The Metalation of Phenoxathiin". J. Am. Chem. Soc, 62(10), 2606-2611, 1940.

[3] Suter. C. M. and Maxwell. C. E. "Organic Syntheses". (R. C. Fuson, Editor), John Wiley and Sons, Inc, New York, Vol. 18, pp. 64, 1938.

[4] Zhao. B. J, Shine. H. J, Marx. J. N, Hofmann. C. and Whitmire. K. H. "Addition of the Phenoxathiin Cation Radical to Alkenes and Nonconjugated Dienes. Formation of $(E)$ - and $(Z)-(10-$ Phenoxathiiniumyl) alkenes and $(E)$ - and (Z)-(10-Phenoxathiiniumyl) dienes on Basic Alumina". J. Org. Chem, 72(16), 61546161, 2007.

[5] Radutiu. A. C, Baciu. I, Caproiu. M. T, Draghici. C, Nicolae. A, Constantinescu. T. and Balaban. A. T. "2-( $\alpha$-aryloxyacetyl)phenoxathiin derivatives". Rev. Roum. Chim, 51(7-8), 653-661, 2006.

[6] Ionescu. S. and Popovici. D. "2Phenoxathiinyl-5-phenyloxazole and 5-phenoxathiinyl-2-phenyloxazole derivatives: Experimental and theoretical study of emission properties". SpectrochimicaActa Part A: Molecular and Biomolecular Spectroscopy, 66 (4-5), 11651170, 2007.

[7] Aly. A. A and Wasfy. A. A. F. " $\gamma-$ Oxocarboxylic Acids in Heterocyclic Synthesis IV. Synthesis of Some Pyridazines Containing Phthalyl- and Tosylamino Acids Using Dicyclohexylcarbodiimide as the Condensing Agent". Chem. Pap, 58(2),126132, 2004.

[8] Ueda. M, Aizawa. T. and Imai. Y. "Preparation and properties of polyamides and polyimides containing phenoxathiin units".Journal of Polymer Science Part A: Polymer Chemistry, 15(11),2739-2747, 1977.
[9] Tintaru, A, Hillebrand, M. and Thevand. A. "NMR Study of the inclusion complexes of carboxy-phenoxathiin derivatives with cyclodextrin". Journal of Inclusion Phenomena and Macrocyclic Chemistry, 45(1-2), 35-40, 2003.

[10] Aura. T, Melania. O, Daniela. G. and Mihaela. H. "Experimental and Theoretical Study of the Ground and Excited State Properties of 3-Carboxyphenoxathiin". Rev. Roum. Chimie, 49(3-4), 317-326, 2004.

[11] Yas. M, Foubelo. F. and Ferrandez. J. V. "Dibenzothiepins, phthalans and phthalides from 4-heterosubstituted dibenzothiins". Tetrahedron, 59(12), 2083-2092, 2003.

[12] Mauthner. F. "Ueber das Phenoxthin und Naphtoxthin". Ber, 39(2), 1340-1347, 1906.

[13] Bennett. G. M, Lesslie. M. S. and Turner. E. E. "The configuration of heterocyclic compounds. Part V. Thianthren and phenoxthionine derivatives", J. Chem. Soc, 37, 444-446, 1937.

[14] Suter. C. M, McKenzie. J. P. and Maxwell. C. E. "Phenoxthin. I. A Comparison of the Directive Influences of Oxygen and Sulfur". J. Am. Chem. Soc, 58(5), 717-720, 1936.

[15] Varun. A, Pragi. A. and Lamba. H. S. "Synthesis and evaluation of chalcone derivatives of 2-acetyl naphthalene for antifungal and antibacterial activity". Der Pharmacia Lettre, 4(2), 554-557, 2012.

[16] Kapubalu. S. K, Kovvuri. T. R, Appikonda. V, Gudaparthi O. and Dubeyb. P. K. "Synthesis and characterization of some novel isoxazoles via chalcone intermediates". Der PharmaChemica, 3(5), 113-122, 2011.

[17] Shantaram. K, Popat. M, Ramdas. P. and Appala. R. "Synthesis and pharmacological evaluation of isoxazole derivatives containing 1,2,4-triazole Moiety". Marmara Pharmaceutical Journal, 16(2), 134-140, 2012. 


\section{الخلاصة}

الهـدف مــن هــذا العمـل هـــو تحضــير مشــتقات

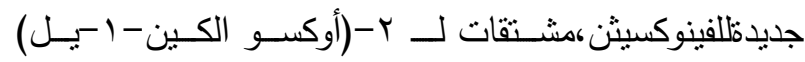

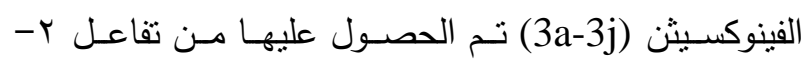

أسيتيل فينوكسيثن مع مختلف المركبات العطريـة الالدهايديـة

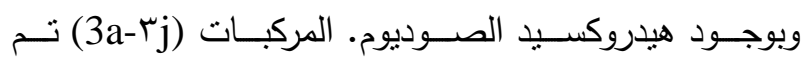

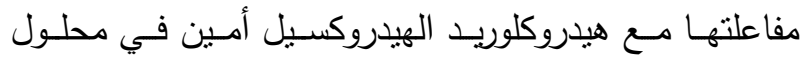

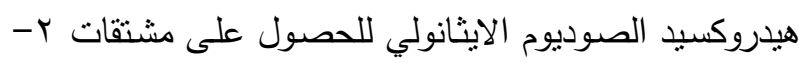

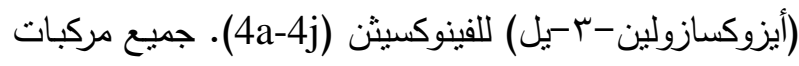

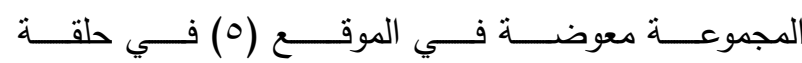

الايزواوكسـازولين بمجـاميع أريـل وحسـب المركبـات العطريـة

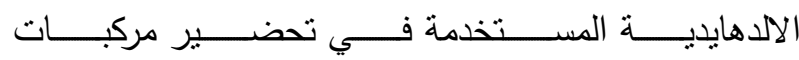

(3a-3j). كل المركبات المحضـرة شخصت بتثنيات مختلفة

لتاكيد التراكيب التي تم الحصول عليها. 